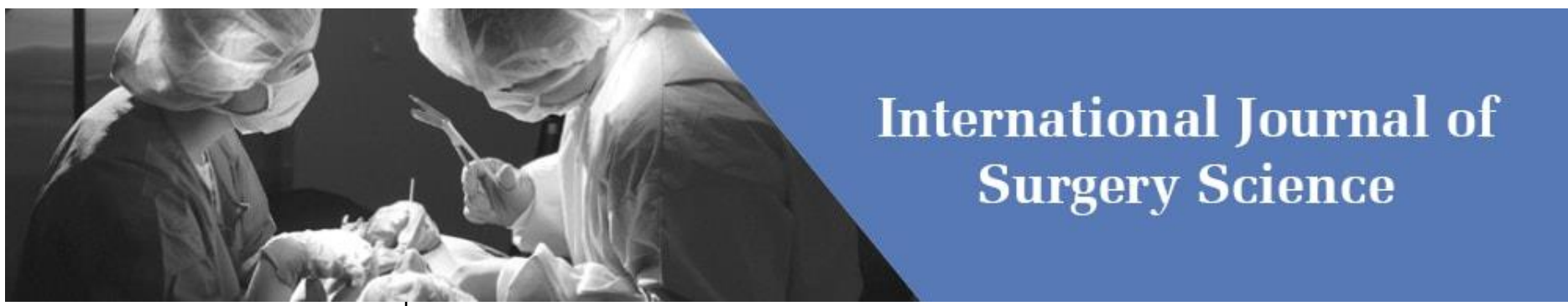

E-ISSN: 2616-3470

P-ISSN: 2616-3462

(C) Surgery Science

www.surgeryscience.com

$2019 ; 3(3): 186-189$

Received: 01-05-2019

Accepted: 03-06-2019

Dr. Avinash Kurudi Siddappa

Assistant Professor of Surgery,

SSIMS \& RC, Davangere,

Karnataka, India

Dr. Suresh U Kadli

Assistant Professor of Surgery,

Basaveshwara Medical College,

Chitradurga, Karnataka, India

Dr. Kailas CT

Assistant Professor of Surgery, JJM Medical College, Davangere,

Karnataka, India

\section{Dr. RL Chandrasekhar}

Professor \& Head of Surgery,

JJM Medical College, Davangere,

Karnataka, India

\section{Correspondence}

Dr. Suresh U Kadli

Assistant Professor of Surgery,

Basaveshwara Medical College,

Chitradurga, Karnataka, India

\section{A study on management of multinodular goiter at a tertiary care hospital}

\author{
Dr. Avinash Kurudi Siddappa, Dr. Suresh U Kadli, Dr. Kailas CT and \\ Dr. RL Chandrasekhar
}

DOI: https://doi.org/10.33545/surgery.2019.v3.i3d.166

Abstract

Worldwide, nodular goitre remains a problem of enormous magnitude affecting less than 5\% of the population. Neither a well formulated nor a simple procedure is available for the management of MNG. This was a prospective study of 50 cases of MNG with an adequate follow up to look for post op complications. FNAC was done all patients and was found to be $100 \%$ accurate. Majority of the patients were treated with STT, followed by HT and NTT. Complications included wound infection and RLN palsy. FNAC is an invaluable and highly accurate tool in the diagnosis of MNG. Surgery, STT was found to be an adequate and safe treatment for non-toxic MNG with minimal complications.

\section{Keywords: MNG, FNAC, Goitre, STT, NTT, HT}

\section{Introduction}

Though the thyroid and its diseases were known to the physicians from the time immemorial, the subject still continues to evince great interest even today from the clinician and the pathologist alike. As Willis stated, for the thyroid gland, working in continuous state of interest having alternate periods of cellular activity and quittance in response to an array of exogenous and endogenous stimuli, it is not so surprising that it suffers from a wide variety of disease. A constant supply of thyroid hormones is necessary for growth, development and maintenance of metabolism and functional activity of most organs. Diseases of thyroid are manifested either qualitative and quantitative alterations in hormone secretion or enlargement of thyroid (goitre) or both. ${ }^{[1]}$

The thyroid gland, reddish brown and highly vascular, is placed anteriorly in the lower neck. Level extends from the $5^{\mathrm{th}}$ cervical to the $1^{\mathrm{s}} \mathrm{t}$ thoracic vertebra. Its weight is usually 25 grams. The gland is slightly heavier in the female sex and enlarges during pregnancy and menstruation. [1] The thyroid gland functions primarily to produce thyroid hormones. It stimulates the oxygen consumption of most cells in the body, helps regulate lipid and carbohydrate metabolism and are necessary for normal growth and maturation. ${ }^{[2]}$

Nodular goitre is probably the most common endocrine problem in the world today.

The commonly accepted definition of Goitre "A Thyroid Gland i.e., at least twice its normal size". In general, the term is applied to the benign enlargement of thyroid gland. ${ }^{[3]}$

Worldwide, nodular goitre remains a problem of enormous magnitude. It is estimated that no less than $5 \%$ of the world's population have goitres. Depending on the population studied, multinodular goitre occurs in upto $12 \%$ of adults. Multinodular goitre is more common in women than men and increases in prevalence with age. The incidence of carcinoma in multinodular goitre has been reported as $5 \%$ to $10 \%$. ${ }^{[4]}$

Neither a well formulated nor a simple procedure is available for the management of patients with MNG. The main reason for such situation to exist is, nodular lesions may represent one of the many different cell types that cannot be distinguished from one another without histologic study. These nodules may be benign or malignant. Ultimately, it is this threat of malignancy that poses a major problem. 
FNAC is a diagnostic tool in which cells are extracted from the palpable swelling using FNAC gun, syringe and fine needle. It is simple, quick, safe and cost- effective. FNAC is increasingly being used for evaluation of thyroid swellings.

The study of multinodular goitre is essential, as it is a commonly encountered endocrine problem in clinical practice. The major concern in modern thyroid gland surgery is morbidity. Besides haemorrhage and hypoparathyroidism, damage to the recurrent laryngeal nerve is the complication most feared by both patient and surgeon. It represents a serious complication inducing, when bilateral, serious functional sequalae such as phonatory, respiratory and psychological problems that limit working capacities and social relationships of the patients. Post- operative respiratory complications may need either lifesaving endotracheal intubation followed by tracheostomy or immediate tracheostomy. ${ }^{[5]}$

\section{Methodology}

This is a randomized prospective clinical study of multinodular goitre patients admitted in General Hospital, attached to Medical Collage. A total number of 50 cases studied in a span of 24 months. Complete clinical examination, and necessary

laboratory investigations were performed.

\section{Inclusion criteria}

Patients with enlargement of thyroid gland, with more than one nodule palpable or enlarged thyroid gland with nodular surface. Both toxic and non-toxic multinodular goitres were included in the study.

\section{Exclusion criteria}

Diffuse hyperplastic goitre, Solitary nodule of thyroid, Thyroid enlarge me nt with the clinical features suggestive of malignancy and multinodular goitre patients not undergoing surgery were excluded.

The pre- operative treatment in most cases consisted of supportive treatment to control of toxicity in cases of toxic multinodular goitre. Patients operated after getting medical fitness for surgery. The operated specimen was sent for histopathologic examination in all cases. Movement of vocal cords were noted at the end of the operation in every case.

\section{Results}

Table 1: Distribution of secondary thyrotoxicosis

\begin{tabular}{|c|c|c|c|c|}
\hline Type of goiter & \multicolumn{2}{|c|}{ Number of cases } & Total & Percentage \\
\hline & Male & Female & & \\
\hline Nontoxic multinodular goitre & 3 & 41 & 44 & 88 \\
\hline Toxic multinodular goiter & 0 & 6 & 6 & 12 \\
\hline
\end{tabular}

Out of 50 cases, 44 cases (88 percent) were nontoxic multinodular goitre and 6 (12 percent) were toxic multinodular goitre Fine needle aspiration cytology was done in all cases. Majority reported as nodular colloid goitre, 2 cases as Hashemite's thyroiditis and 1 case had a nodular goitre with cystic changes.

Indirect laryngoscopy was done in all the cases, and bilateral vocal cord movement was normal.

Thyroid function tests (T3, T4, and TSH) were done in all patients. In 6 cases, thyrotoxicosis was confirmed by thyroid function tests.

Sleeping pulse rate were within normal limits in all patients except in 6 who had secondary thyrotoxicosis.

All cases were operated. Surgery was advocated for reasons of cosmos, pressure symptoms and secondary thyrotoxicosis. In toxic cases, the toxicity was controlled with carbimazo le $10 \mathrm{mg}$, 3- 4 times daily for a period ranging from 4- 12 weeks and Propranolol $40 \mathrm{mg}, 3$ times daily along with carbimazole.

Table 2: Types of Surgery done

\begin{tabular}{|c|c|c|}
\hline Types of Surgery & Number of Cases & Percentage \\
\hline Subtotal thyroidectomy & 37 & 74 \\
\hline Hemithyroi dectomy & 7 & 14 \\
\hline Near total thyroidectomy & 5 & 10 \\
\hline Total thyroidectomy & 1 & 2 \\
\hline
\end{tabular}

Subtotal thyroidectomy was done in 37 cases (74 percent) followed by Hemithyroi dectomy 7 cases $(14 \%)$, Near total thyroidectomy 5 cases $(10 \%)$ and total thyroidectomy 1 case ( $2 \%$ ).

Table 3: Postoperative Complications

\begin{tabular}{|c|c|c|}
\hline Type of Complications & Number of Cases & Percentage \\
\hline Recurrent laryngeal nerve palsy (bilateral) & 1 & 2 \\
\hline Parathyroid insufficiency & 0 & 0 \\
\hline Wound infection & 2 & 4 \\
\hline Mortality & 0 & 0 \\
\hline
\end{tabular}

No pre-operative and per operative complications occurred in the present series. Recurrent laryngeal nerve palsy (bilateral) was found in 1 case ( 2 percent), which was transient and recovered after 1 month. There is no parathyroid insufficiency Wound infection in 2 case (4 percent) was the other complication found in the present study. No mortality occurred in the present study.
According to histopathological analysis in present study of 50 cases, 37 cases (76 percent) showed features of benign goiter, toxic goitre showed sec toxic changes in 6 cases (12 percent), 7 Hashimoto's thyroiditis.

Follow up

Follow up ranged from 2 months to 24 months. 
All 7 cases of Hashimoto's thyroiditis and majority of other cases who underwent subtotal thyroidectomy were put on tablet Levothyroxine $0.15 \mathrm{mg}$ once daily.

\section{Discussion}

FNAC was found to be very useful in the evaluation of MNG. In majority of the cases where the FNAC was benign it proved to be benign $\mathrm{MN} G$ on postoperative histopathological examination.

This shows that FNAC is $100 \%$ accurate. FNAC is an invaluable, minimally invasive, highly accurate and cost effective procedure for pre-operative assessment of patients with thyroid lesions.

One of the ca ses was reported as thyroid cyst on ultrasound on scan and it was finally diagnosed as MNG.

Patient with vocal cord palsy diagnosed on indirect laryngoscopic examination and positive neck lymph nodes associated with thyroid swelling were excluded in this study.

The main indication for surgery in our series was the swelling itself either due to cosmetic reasons or fear of malignancy on the part of the patient. Subtotal thyroidectomies were done 37 (74\%) cases, Hemithyroi dectomy in $7(14 \%)$ near total $5(10 \%)$ and total thyroidectomy in $1(2 \%)$ were carried out when there was high suspicion of malignancy.

We encountered no complication of surgery, apart from irritation of the throat is early postoperative periods. Which subsided without any active management. The average duration of stay in the hospital was 8 days.

According to histopathological analysis in the presentstudy of 50 cases, $37(74 \%)$ cases showed features of benign goitre, 7 (14\%) cases of Hashimoto's thyroiditis. and 6 (12\%) case of toxic multinodular goitre.

Rao and Reddy reported 7\% distribution of Hashimoto's thyroiditis and Ahuja reported 9\%. The present study with $14 \%$ is comparable to above studies.

The most important investigation of thyroid function is meticulous clinical assessment of the patient. But clinical diagnosis has to be confirmed by investigate ions to know exactly the hormonal status of the thyroid and also its relation with the anterior pituitary and hypothalamus.

There are a variety of tests of thyroid function available. The number of investigations requested should be the minimum necessary to reach a diagnosis and formulate a management plan. Only a small number of parameters needs to be measured as a routine although this may require supplementation or repeat when inconclusive. ${ }^{[6-7]}$

The serum TSH may be used as a single, initial test of thyroid function because of the high sensitivity of the TSH assay to diagnose both hyperthyroidism and hypothyroidism. TSH levels can be measured accurately down to very low serum concentrations and if the serum TSH level is in the normal range it is redundant to measure the $\mathrm{T}_{3}$ and $\mathrm{T}_{4}$ levels. Interpretation of deranged TSH levels however depends on knowledge of the $\mathrm{T}_{3}$ and $\mathrm{T}_{4}$ values. ${ }^{[8]}$

In the eruthyroid state, $\mathrm{T} 3, \mathrm{~T}_{4}$ and $\mathrm{TSH}$ levels will all be within the normal range. Florid thyroid failure results in depressed $\mathrm{T}_{3}$ and $\mathrm{T}_{4}$ levels with gross elevation of the $\mathrm{TSH}$. Incipient or developing thyroid failure is characterized by low normal values of $\mathrm{T}_{3}$ and $\mathrm{T}_{4}$ and elevation of the TSH. In toxic states the TSH level is suppressed and undetectable. ${ }^{[9]}$

Thyroxine $\left(\mathrm{T}_{4}\right)$ and tri -iodothyronine $\left(\mathrm{T}_{3}\right)$ are transported in plasma bound to specific proteins (thyroxine - binding globulin, TBG). Only a small fraction of the total $\left(0.03\right.$ per cent of $\mathrm{T}_{4}$ and 0.3 percent of $\mathrm{T}_{3}$ ) is free and physiologically active. Assays of both total and free hormone are available but the total values depend on the level of circulating proteins which are affected by the level of circulating oestrogen. Thus, pregnant women and those on the oral contraceptive pill have elevated total $\mathrm{T}_{4}$ and $\mathrm{T}_{3}$ levels without evidence of toxicity. The free hormone levels are unaffected. Similarly some patients have low levels of TBG either as a primary phenomenon or secondary to a reduction in serum protein levels as a result of systemic or liver disease and the total level of circulating hormone may be low. For these reasons the free levels are more meaningful. Highly accurate radioimmunoassay of free $T_{3}$ and $T_{4}$ are now routine. $T_{3}$ toxicity (with a normal $\mathrm{T}_{4}$ ) is a distinct entity and may only be diagnosed by measuring the serum $\mathrm{T}_{3}$, although a suppressed TSH level with a normal $\mathrm{T}_{4}$ is suggestive, are useful in detecting hypothyroidism or hyperthyroidism, but the majority of patients with thyroid nodules are eu thyroid. ${ }^{[1-8]}$ the majority of patients with thyroid no dules are eu thyroid. ${ }^{[10]}$

It gives information about thyroid activity and also shows graphic information about the size and extent of the gland. This method is particularly helpful in showing the retrosternal extent of the gland.

The uptake by the thyroid of a low dose of either radiolabeled iodine ( $\mathrm{I}^{123}$ ) or tecnnetium- $99 \mathrm{~m}$ (Tc ${ }^{99} \mathrm{~m}$, which is normally taken up like $\mathrm{I}^{123}$ ) will demonstrate the distribution of activity in the whole gland. This test is inappropriate for distinguishing benign from malignant les ions because the majority ( 80 per cent) of 'cold' swellings is benign and some (5 per cent) functioning or 'warm' swellings will be malignant. Its principal value is in the toxic patient with a nodule or nodularity of the thyroid. Localization of over- activity in the gland will differentiate between a toxic nodule with suppression of the remainder of the gland and toxic multi nodular goitre with several areas of increased uptake with important implications for therapy. Routine isotope scanning is unnecessary. ${ }^{[11-12]}$

\section{Conclusion}

FNAC is an invaluable, minimally invasive. Highly accurate and cost effective procedure for preoperative assessment of patients with thyroid lesions.

The main indications for surgery in MNG are cosmetic problem and Subtotal thyroidectomy is the ideal treatment of MNG. FNAC had very large correlation. Whereas Hashimoto's thyroiditis, were negatively correlated as per the GoodmanKruskal Gamma coefficient. FNAC is very useful in the diagnosis and management of MNG.

\section{References}

1. Tseng YC, Burman KD, Schudies RP et al. Effects of epidermal growth factors on thyroglobulin and adenosine 3'5'-mono phosphate production by cultured human thyrocytes, J Clin Endocrinol Metab. 1989; 69:771.

2. Grube Lobenstein $\mathrm{K}$, Buchan $\mathrm{G}$, Sadeghi $\mathrm{R}$ et al. Transforming growth factor beta-regulates thyroid growth role in the pathogenesis of nontoxic goiter. J Clin Invest. 1989; 83:764.

3. Tan GH, Gherib H. Reading solitary thyroid nodule Comparison between palpation and USG, Arch int med. 1995, 155, 2418-2423.

4. Katoh R, Bray CE, Suzuki K, Komiyama A, Hemmi A, Kawaoi A et al. Growth activity in hyperplastic and neoplastic human thyroid determined by a immuno histochemical staining procedure using monoclonal antibody MIB-1, Human pathology. 1995; 26:139-146.

5. Farquharson N, Moran B. Surjery of the neck, ch 9, 
Farquharson's text book opertive general surgery 9th ed. Edward Arnold, 2003, 155-178.

6. Das S. The thyroid and parathyroid in A consice textbook of surgery, 3rd ed, calcutta, 2004, 642-676.

7. Are C, Zeiger MA. Thyroid nodule in Current surgical therapy,Cameron JL 8th ed, Mosby, 2004, 58-82.

8. Das S. Examination of the thyroid gland, ch 27, A manual on clinical surgery, 6th ed, Calcutta, India, 2005, 284-297.

9. Woeber Kenneth A, Cost -effective evaluation of the patient with a thyroid nodule, Surg Clin North Am. 1995; 75:357363.

10. Abel nabi H, Falko JM, Olsen. Solitary cold Thyroid nodule: Cost and effectiveness of USG, South Med J. 1984; 77:1146-1148.

11. Tan GH, Gherib H. Reading solitary thyroid nodule Comparison between palpation and USG, Arch int med. 1995; 155:2418-2423.

12. Rodriguez JM, Parilla P, Sola J, Bes A, Aguliar J, Moreno A et al. Comparison between preoperative cytology and intra operative frozen section biopsy in diagnosis of thyroid nodule, Br J sur. 1994; 81:1151-1154. 